\title{
Asymmetry Coefficients as Indicators of Chaos
}

\author{
P. WȦ̧̇ $\dot{Z}^{a, *}$ AND D. BIELIŃSKA-W Ȧ̧̇ $\dot{Z}^{b}$ \\ ${ }^{a}$ Centrum Astronomii, Uniwersytet Mikołaja Kopernika, Gagarina 11, 87-100 Toruń, Poland \\ ${ }^{b}$ Instytut Fizyki, Uniwersytet Mikołaja Kopernika, Grudziądzka 5, 87-100 Toruń, Poland
}

(Received August 11, 2008)

\begin{abstract}
The aim of this paper is to present a new simple indicator of chaos derived from the dynamics of the motion. For this purpose statistical methods are used. A function describing the motion of the analyzed system (in the example under consideration, the time dependence of the angle of a damped driven pendulum, $\omega(t))$ is recorded in time intervals $t \in\left\langle T_{\mathrm{s}}, T_{f_{k}}\right\rangle, k=1,2, \ldots \mathrm{K}$, with $T_{f_{k}}>T_{f_{k-1}}$. Each of the recorded functions is considered as a statistical distribution. The asymmetry coefficients of the set of distributions form a series and their behavior in periodic and chaotic regions is compared. It is shown that the behavior of this series in the chaotic and in the periodic regimes is entirely different. The changes of the asymmetry coefficients for the periodic cases are very regular and for the chaotic ones - random. In periodic cases, the coefficients converge to zero when the length of the distribution increases.
\end{abstract}

PACS numbers: 02.70.Rr, 95.10.Fh, 05.45.-a, 05.45.Gg, 05.45.Tp

\section{Introduction}

The distinction between chaotic and periodic behavior of dynamical systems may be performed using different kinds of criteria [1-4]. An excellent review of the properties of chaotic systems and a description of a variety of indicators, may be found in the book by Gutzwiller [5]. The most commonly known indicators are the Lyapunov exponents $[6,7]$. The control of chaotic systems can also be performed via time-series analysis [8]. An efficient method of chaos detection (the relative Lyapunov indicator) has been proposed in [9]. Other interesting investigations have been presented by Skokos et al. [10]. The authors introduced quantities that clearly distinguish between the chaotic behavior and the quasiperiodic motion on $N$-dimensional tori (the generalized alignment index, GALI). The application and efficient computational technique of GALI indices has also been shown by the authors in [11].

Usually only a limited set of the observational data is available, and the task of determining whether the observed motion is chaotic or periodic may be difficult and the uncertainty of the results rather large. The best known and the most spectacular application of such an analysis is determining whether a hypothetical planetary system is stable and, as a consequence, whether the hypothesis that the system exists is valid [12,13]. In such cases the availability of simple and reliable indicators of chaos is of a particular importance.

In this work we propose alternative, very simple and

\footnotetext{
* corresponding author; e-mail: Piotr.Waz@astri.uni.torun.pl
}

related to the observational data, statistical indicator of chaos. The indicator has been obtained from an analysis of the statistical behavior of an ensemble derived from the time dependence of selected quantities characterizing the motion: we analyze the shapes of functions $\omega(t)$ describing the time dependence of the angle of a pendulum. In the analysis, the functions are treated as statistical distributions characterized by their moments. The behavior of the moments (in particular the asymmetry coefficients) depends on the character of the motion: In the chaotic regime it is different than in the periodic one.

Considering functions describing different kinds of physical processes as statistical distributions characterized by some parameters related to different aspects of their shapes is commonly used in many areas of physics and leads to a variety of very useful methods. In particular, the principle of moments according to which the degree of similarity of a pair of distributions is determined by the number of the lowest statistical moments which are equal for both distributions [14] has recently been applied by the present authors in the theory of molecular similarity $[15,16]$ and in astrophysics [17]. Studies on the shapes of the intensity distributions of spectra resulted in the so-called statistical theory of spectra [18-20]. Also a method of determining the envelopes of molecular electronic bands [21] or statistical studies on properties of spectra of the Heisenberg Hamiltonian [22] may be mentioned in this context.

\section{Method}

Let $\Omega(t)$ be a function characterizing the motion we are going to analyze. It may be the time dependence of the angle of a pendulum, of the velocity or the position of a 
planet, etc. Let us define a time-series $\Omega_{k}(t) \equiv\{\Omega(t), t \in$ $\left.\left\langle T_{\mathrm{s}}, T_{f_{k}}\right\rangle\right\}, k=1,2, \ldots K$ with a fixed $T_{\mathrm{s}}$ and $T_{f_{1}}<$ $T_{f_{2}}<\ldots T_{f_{\mathrm{K}}}$. The terms of the series are characterized by the time intervals $\Delta T_{k}=T_{f_{k}}-T_{\mathrm{s}}$ and are treated as statistical distributions. The starting time $T_{\mathrm{s}}$ and the final one $T_{f_{k}}$ denote the beginning and the end of the $k$-th distribution $\Omega_{k}(t)$ and $\Delta T_{k}$ is its length. The asymmetry coefficients of the distributions read

$$
\begin{gathered}
M_{q}^{\prime \prime}\left(k, \Delta T_{k}\right)=\mathcal{N}\left(k, \Delta T_{k}\right) \int_{T_{\mathrm{s}}}^{T_{f_{k}}}\left(\Omega_{k}(t)+c\right) \\
\quad \times\left[\frac{t-M_{1}\left(k, \Delta T_{k}\right)}{\sqrt{M_{2}\left(k, \Delta T_{k}\right)-M_{1}\left(k, \Delta T_{k}\right)^{2}}}\right]^{q},
\end{gathered}
$$

where

$$
\begin{aligned}
& \mathcal{N}\left(k, \Delta T_{k}\right)=\left[\int_{T_{\mathrm{s}}}^{T_{f_{k}}}\left(\Omega_{k}(t)+c\right) \mathrm{d} t\right]^{-1}, \\
& M_{n}\left(k, \Delta T_{k}\right)=\mathcal{N}\left(k, \Delta T_{k}\right) \int_{T_{\mathrm{s}}}^{T_{f_{k}}}\left[\Omega_{k}(t)+c\right] t^{n} \mathrm{~d} t,
\end{aligned}
$$

$n=0,1,2,3, \ldots, q=2 j+1, j=1,2,3, \ldots$ and $c$ is a numerical constant.

For a totally symmetric distribution $M_{q}^{\prime \prime}$ are equal to zero. Generally, $M_{q}^{\prime \prime}$ can be negative or positive depending on the direction of the asymmetry.

Usually, in practical applications, $\Omega(t)$ is known from experimental measurements. Then, its values are given in a discrete set of points $\left\{\tau_{i}\right\}$. The asymmetry coefficients of the discrete $k$-th distribution $\Omega_{t^{k}}$ read

$$
\begin{aligned}
& M_{q}^{\prime \prime}\left(k, N_{k}\right)=\mathcal{N}\left(k, N_{k}\right) \\
& \quad \times \sum_{i=1}^{N_{k}}\left(\Omega_{t_{i}^{k}}+c\right)\left[\frac{t_{i}^{k}-M_{1}\left(k, N_{k}\right)}{\sqrt{M_{2}\left(k, N_{k}\right)-M_{1}\left(k, N_{k}\right)^{2}}}\right]^{q},
\end{aligned}
$$

where

$$
\begin{aligned}
& \mathcal{N}\left(k, N_{k}\right)=\left[\sum_{i=1}^{N_{k}}\left(\Omega_{t_{i}^{k}}+c\right)\right]^{-1}, \\
& M_{n}\left(k, N_{k}\right)=\mathcal{N}\left(k, N_{k}\right) \sum_{i=1}^{N_{k}}\left(\Omega_{t_{i}^{k}}+c\right)\left(t_{i}^{k}\right)^{n},
\end{aligned}
$$

$n=0,1,2,3, \ldots, q=2 j+1, j=1,2,3, \ldots$ and $c$ is a numerical constant. $N_{k}$ is the number of measurement points in the $k$-th distribution, i.e. $t_{i}^{k}=\tau_{i}$, $i=1,2, \ldots N_{k}, k=1,2, \ldots K$, with $t_{1}^{k}=T_{\mathrm{s}}, t_{N_{k}}^{k}=T_{f_{k}}$ and $N_{1}<N_{2}<\ldots<N_{K}$ (i.e. $T_{f_{1}}<T_{f_{2}} \ldots<T_{f_{K}}$ ). Since $T_{\mathrm{S}}$ is the same for all $k$, the length of the $k$-th distribution is proportional to $N_{k}$ (or to $T_{f_{k}}$ ).

The qualitative results are the same for all values of $c$, as it will be shown in the subsequent chapter. However, as it is in the theory of statistics, we have to operate with positive distributions. Therefore we assume that the conditions $\Omega_{k}(t)+c \geq 0$ or $\Omega_{t_{i}^{k}}+c \geq 0$ are fulfilled for continuous and discrete distributions, respectively.

As it will be shown, the dependence of the asymmetry coefficients (the skewness $M_{3}^{\prime \prime}$ and the higher order asymmetry effects described by $M_{5}^{\prime \prime}$, and $M_{7}^{\prime \prime}$ ) on the lengths of the distributions, in the periodic cases is essentially different than in the chaotic ones. This difference of the behavior is understandable: The periodic motion is, by definition, self-similar over sufficiently large intervals of time. The chaotic one does not exhibit, also by definition, any self-similarity. On the other hand, taking a constant value of $T_{\mathrm{S}}$ and a series of increasing values of $T_{f}$, we can check how the asymmetry changes when the lengths of the distributions increase. In this way we analyze the self-similarity of $\Omega(t)$. We expect, and this expectation is confirmed by the example discussed in the following section, that for the periodic motion the asymmetry coefficients approach 0 while $T_{f}$ (or the number of measurement points, $N$, in a discrete case) approaches infinity. In the case of chaos no regular asymptotic behavior is expected.

\section{Results and conclusions}

Let us consider a damped driven pendulum. According to the Newton second law, the equation of motion for the pendulum of mass $m$ and length $l$ can be written as

$$
m l^{2} \frac{\mathrm{d}^{2} \theta}{\mathrm{d} t^{2}}+\gamma \frac{\mathrm{d} \theta}{\mathrm{d} t}+m g l \sin \theta=A \cos \left(\omega_{\mathrm{D}} t\right) .
$$

The lhs terms represent acceleration, damping, and gravitation. The rhs term is the driving force.

The equation of motion (7) can be written as a system of three first order equations [23]:

$$
\left.\begin{array}{rl}
\frac{\mathrm{d} \omega}{\mathrm{d} t} & =-(1 / d) \omega-\sin \theta+g \cos \phi, \\
\frac{\mathrm{d} \theta}{\mathrm{d} t} & =\omega, \\
\frac{\mathrm{d} \phi}{\mathrm{d} t} & =\omega_{\mathrm{D}},
\end{array}\right\}
$$

where $\omega(t)$ is the angle of the pendulum, $d$ is the damping parameter, $g$ is the forcing amplitude, and $\omega_{\mathrm{D}}$ is the angular driving force. The variable $\phi$ is the phase of the driving term. The natural frequency for small amplitudes has been set equal to 1 and the time is dimensionless [24]. The terms $\sin \theta$ and $g \cos \phi$ are nonlinear. Chaos can appear for some particular values of $g$.

A detailed analysis has been performed for $d=2$, $\omega_{\mathrm{D}}=2 / 3$, and for four values of $\mathrm{g}: g=1.07, g=1.15$, $g=1.47$, and $g=1.50$. For $g=1.07$ and for $g=1.47$ the solutions are periodic while for $g=1.15$ and for $g=1.50$ they are chaotic [23]. The differential equations (8) have been solved numerically. The main part of the numerical integration code constitutes the procedure RA15 [25]. The calculations have been performed in equidistant points, i.e. $\tau_{i+1}-\tau_{i}=\delta=$ const, for the time series $\left\{\tau_{i}\right\}_{i=1}^{1000}, \tau_{1}=1, \delta=1$. The functions $\omega(t)$ are plotted in Fig. 1 .

The asymmetry coefficients have been evaluated according to Eq. (4) with $\Omega(t)=\omega(t)$ and $c=\pi$. The origin of each distribution corresponds to the initial time $T_{\mathrm{s}}=1$ (fixed for all distributions). The final points (the ends) of the distributions have been selected as $T_{f_{k}}=10 k$, $k=1,2, \ldots 100$. Since $\tau_{1}=1$ and $\delta=1$ in the consid- 


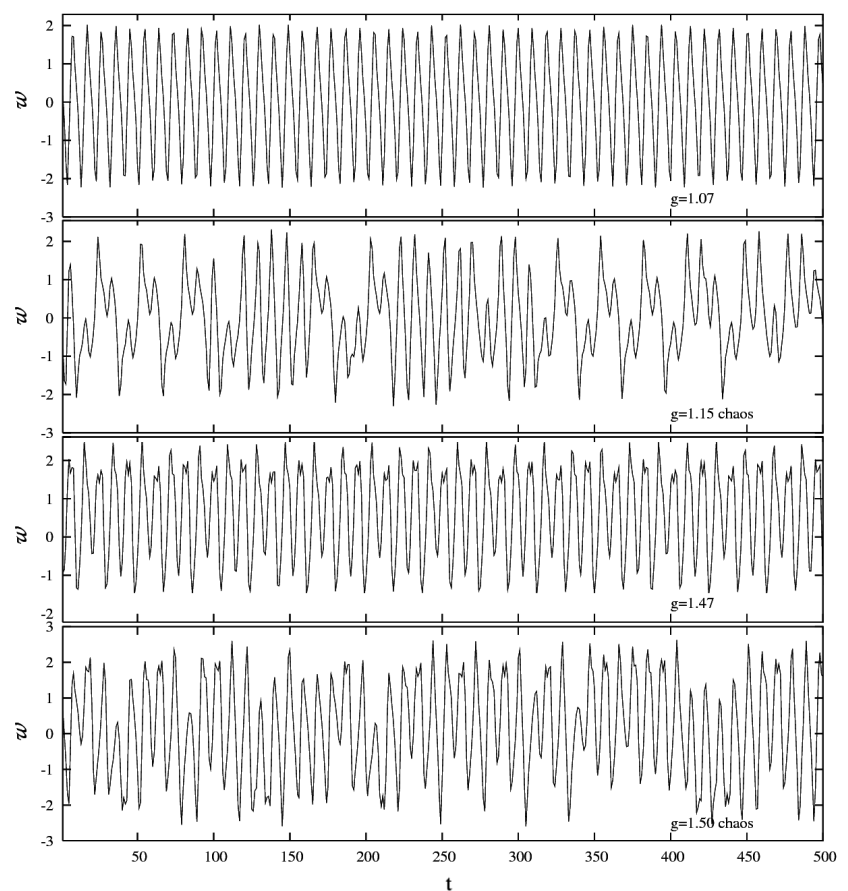

Fig. 1. Functions $\omega(t)$ for the damped driven pendulum for different values of $g$.

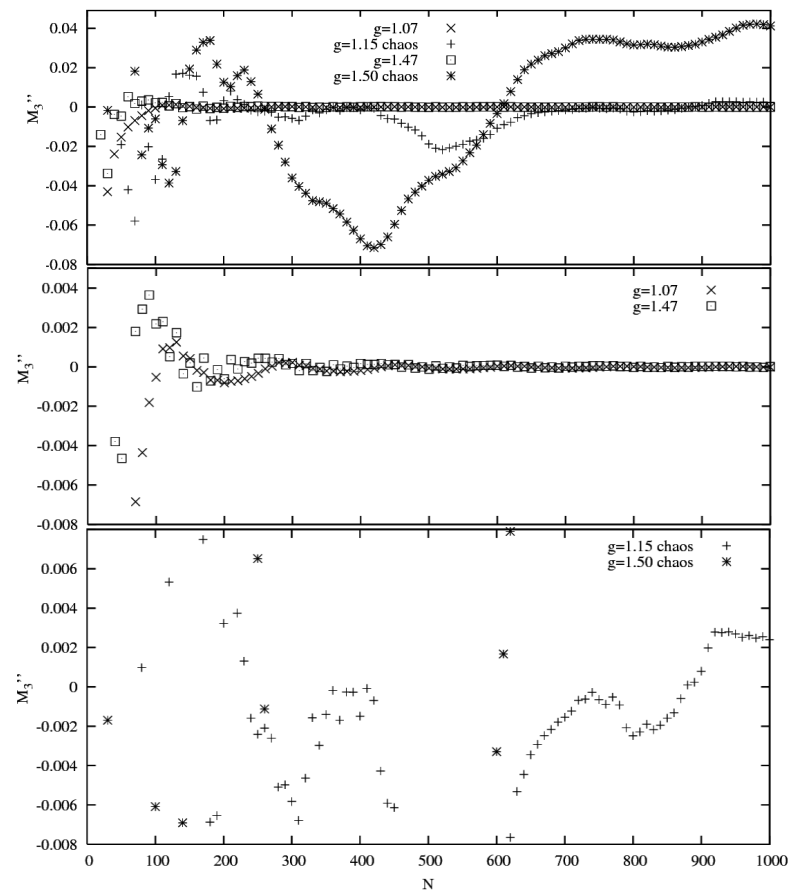

Fig. 2. $\quad M_{3}^{\prime \prime}$ for different values of $g$. The middle and the bottom figures are the enlargements of the top one for the periodic and for the chaotic cases, respectively.

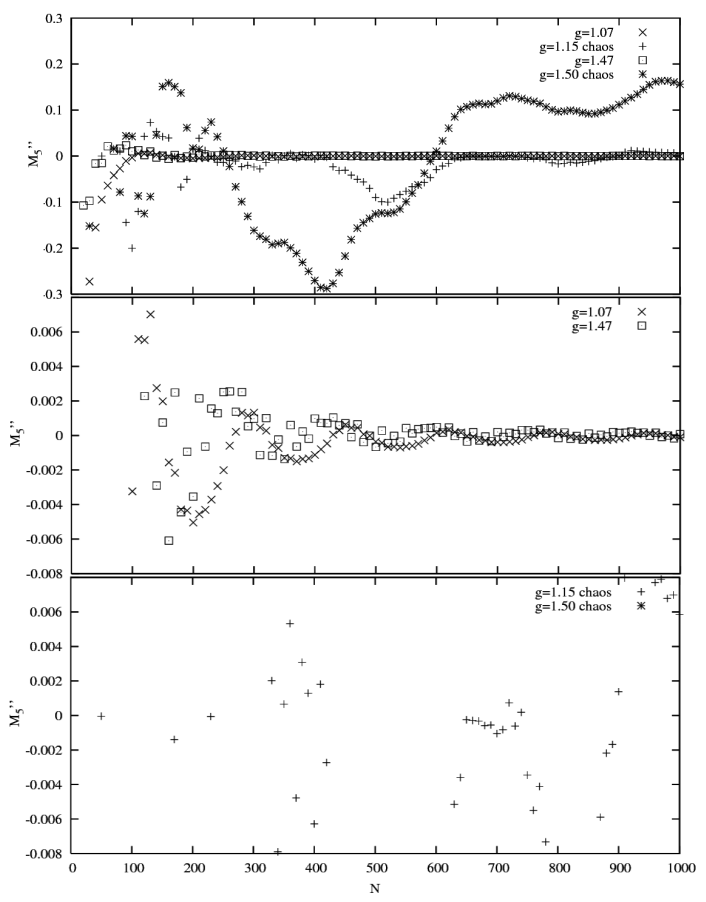

Fig. 3. The same as in Fig. 2, but for $M_{5}^{\prime \prime}$.

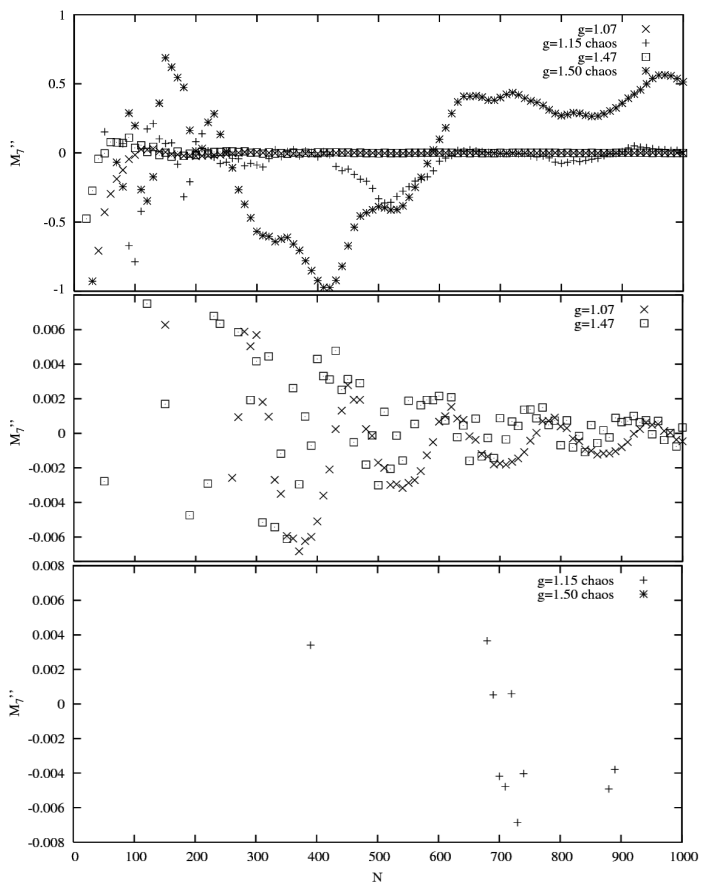

Fig. 4. The same as in Fig. 2, but for $M_{7}^{\prime \prime}$.

ered example, the number of points in the $k$-th distribution $N_{k}=T_{f_{k}}$ and $N_{k}-N_{k-1}=10$.

Figures 2-4 show the asymmetry coefficients $\left(M_{3}^{\prime \prime}, M_{5}^{\prime \prime}\right.$, $\left.M_{7}^{\prime \prime}\right)$ as functions of the lengths of the distributions, i.e. of the number of the measurement points in each $k$-th distribution. The figures consist of three parts. In the 


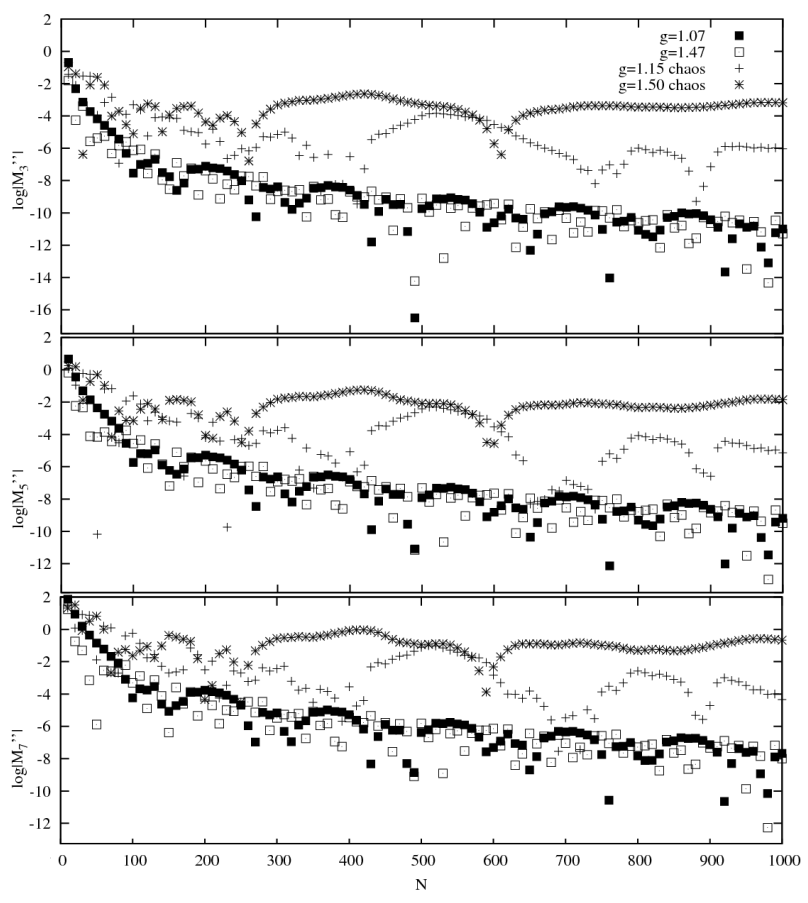

Fig. 5. Logarithms of modules of $M_{q}^{\prime \prime}$. The symbols are the same in all figures and are explained in the upper one.

top sub-figures the ranges of $M_{q}^{\prime \prime}$ have been selected in such a way that all their values fit to the plot. The middle and the bottom sub-figures are the enlargements of the top one. They present, respectively, the periodic and the chaotic cases.

Figure 2 presents the behavior of $M_{3}^{\prime \prime}$. The difference between the periodic and the chaotic motion is clear and obvious. In the periodic cases the changes of the asymmetry coefficients are big only for small values of $N$ (i.e. for short lengths of the distributions for which the periodicity cannot be recognized). For $N \rightarrow \infty$ the asymmetry coefficients converge to 0 (i.e. the distributions converge to the totally symmetric functions). In chaotic cases, the asymmetry coefficients behave in an irregular way. In particular, no signs of convergence to 0 can be seen.

The conclusions derived from Fig. 3, where $M_{5}^{\prime \prime}$ is displayed, and from Fig. 4, which shows the behavior of $M_{7}^{\prime \prime}$, are very much the same. The only new feature is the clearly oscillatory type of convergence.

A convenient way of presentation of these results can be a diagram showing the behavior of the logarithms of $\left|M_{q}^{\prime \prime}\right|$. As one can see in Fig. 5, $\log \left|M_{q}^{\prime \prime}\right|$ converge to large negative values for the periodic cases. In case of chaos there is no convergence and $\log \left|M_{q}^{\prime \prime}\right|$ are larger than for periodic cases.

Another way of using this method can be an analysis of the standard deviations of $M_{q}^{\prime \prime}$ from the mean values, $\sigma\left(M_{q}^{\prime \prime}\right)$. Table shows $\sigma\left(M_{q}^{\prime \prime}\right)$ calculated for $N$ in the range from 800 to 1000 . The qualitative results are the same for any $N$ interval excluding the small $N$ region. The functions $\Omega$ have been shifted to the positive values using different values of $c$. For all $c$ the deviations are much smaller for periodic cases than for the chaotic ones.

Standard deviations $\sigma\left(M_{q}^{\prime \prime}\right)$.

TABLE

\begin{tabular}{c|c|c|c|c|c}
\hline \hline$c$ & $q$ & $g=1.07$ & $g=1.15$ chaos & $g=1.47$ & $g=1.50$ chaos \\
\hline$\pi$ & 3 & 0.000012 & 0.001062 & 0.000012 & 0.013475 \\
& 5 & 0.000074 & 0.005169 & 0.000072 & 0.048207 \\
& 7 & 0.000334 & 0.020628 & 0.000324 & 0.159390 \\
\hline 3.2 & 3 & 0.000012 & 0.001043 & 0.000012 & 0.013236 \\
& 5 & 0.000073 & 0.005074 & 0.000071 & 0.047348 \\
& 7 & 0.000328 & 0.020250 & 0.000319 & 0.156508 \\
\hline 3.4 & 3 & 0.000011 & 0.000981 & 0.000011 & 0.012479 \\
& 5 & 0.000068 & 0.004775 & 0.000067 & 0.044622 \\
& 7 & 0.000309 & 0.019054 & 0.000303 & 0.147383 \\
\hline 3.6 & 3 & 0.000011 & 0.000926 & 0.000011 & 0.011803 \\
& 5 & 0.000065 & 0.004508 & 0.000064 & 0.042193 \\
& 7 & 0.000291 & 0.017991 & 0.000288 & 0.139264
\end{tabular}

Summarizing, the asymmetry coefficients (AC) of the considered distributions are a convenient tool for identifying symptoms of chaos due to simplicity for coding and their clearly different behavior for the chaotic and periodic orbits:

- A smooth convergence to 0 in the periodic cases and an irregular, random distribution of $\mathrm{AC}$ in the case of chaos;

- A convergence to large negative values of $\log |\mathrm{AC}|$ for the periodic cases and no convergence in the case of chaos;

- Close to 0 values of $\sigma(\mathrm{AC})$ for the periodic cases.

\section{Acknowledgments}

Supported by Nicolaus Copernicus University grant No. 417-A.

\section{References}

[1] G. Contopoulos, N. Voglis, Astron. Astrophys. 317, 73 (1997).

[2] C. Froeschlé, E. Lega, Astron. Astrophys. 334, 355 (1998).

[3] Ch. Skokos, J. Phys. A 34, 10029 (2001).

[4] Ch. Skokos, Ch. Antonopoulos, T.C. Bountis, M.N. Vrahatis, J. Phys. A 37, 6269 (2004).

[5] M. Gutzwiller, Chaos in Classical and Quantum Mechanics, Springer Verlag, New York 1990.

[6] G. Benettin, L. Galgani, A. Giorgilli, J.M. Strelcyn, Meccanica 15, 9 (1980); 15, 21 (1980).

[7] A. Wolf, J.B. Swift, H.L. Swinney, J.A. Vastano, Physica D 16, 285 (1985).

[8] C. Piccardi, Chaos 14, 1026 (2004); 16, 043115 (2006), and references therein. 
[9] Z. Sándor, B. Érdi, A. Széll, B. Funk, Celest. Mech. Dyn. Astr. 90, 127 (2004).

[10] Ch. Skokos, T.C. Bountis, Ch. Antonopoulos, Physica $D$ 231, 30 (2007).

[11] Ch. Skokos, T.C. Bountis, Ch. Antonopoulos, Eur. Phys. J. Special Topics 165, 5 (2008).

[12] K. Goździewski, Astron. Astrophys. 398, 1151 (2003).

[13] K. Goździewski, M. Konacki, A. Wolszczan, Astrophys. J. 619, 1084 (2005).

[14] M. Lax, J. Chem. Phys. 20, 1752 (1952).

[15] D. Bielińska-Wa̧ż, P. Wa̧ż, S.C. Basak, Eur. Phys. J. $B$ 50, 333 (2006).

[16] D. Bielińska-Wa̧ż, P. Wạż, S.C. Basak, J. Math. Chem. 42, 1003 (2007).

[17] P. Wąż, D. Bielińska-Wąż, A. Pleskacz, A. Strobel, Acta Phys. Pol. B 39, 1993 (2008).

[18] C.E. Porter, Statistical Theories of Spectra: Fluctuations, Academic, New York 1965.
[19] T.A. Brody, J. Flores, J.B. French, P.A. Mello, A. Pandey, S.S.M. Wong, Rev. Mod. Phys. 53, 385 (1981).

[20] J. Bauche, C. Bauche-Arnoult, M. Klapisch, Adv. At. Mol. Phys. 23, 132 (1988).

[21] D. Bielińska-Wa̧ż, in: Symmetry and Structural Properties of Condensed Matter, Eds. T. Lulek, W. Florek, S. Wałcerz, World Sci., Singapore 1999, p. 212.

[22] D. Bielińska-Wạż, N. Flocke, J. Karwowski, Phys. Rev. B 59, 2676 (1999).

[23] G.L. Baker, J.P. Gollub, Chaotic Dynamics: An Introduction, University Press, Cambridge 1990, 1996.

[24] E.G. Gwinn, R.M. Westervelt, Phys. Rev. A 33, 4143 (1986).

[25] E. Everhart, Celest. Mech. 10, 35 (1974). 